\title{
Noncircular glories and their relationship to cloud droplet size
}

\author{
Philip Laven \\ 9 Russells Crescent, Horley, Surrey, RH6 7DJ, United Kingdom, philip@philiplaven.com
}

Received 13 May 2008; accepted 4 June 2008;

posted 24 June 2008 (Doc. ID 96082); published 11 July 2008

\begin{abstract}
The atmospheric glory caused by backscattering of sunlight from clouds usually has circular colored rings. However, glories with noncircular rings are frequently observed, especially along the edges of clouds. Noting that the angular radius of the rings of glories is a sensitive indicator of the size of the water droplets in clouds, several images of glories have been examined in an attempt to explain the formation of noncircular glories. (C) 2008 Optical Society of America

OCIS codes: $\quad 010.1290,010.1350$.
\end{abstract}

\section{Introduction}

As air travel is now widespread, sightings of atmospheric glories are almost commonplace-at least for those travelers lucky enough to have a window seat! Having taken more than 4000 pictures of glories from commercial aircraft, the author has frequently observed glories with noncircular rings. This paper examines the reasons for the formation of noncircular glories.

The simulations in this paper have been generated using the MiePlot computer program developed by the author. This program can be downloaded free of charge from http://www.philiplaven.com/mieplot.htm.

Previous work $[1,2]$ has shown that the size of water droplets in the clouds can be inferred from images of glories: for example, the glory shown in Fig. 1(a) is compared in Fig. 1(b) with a simulation based on Mie theory calculations for scattering of sunlight by water droplets with radius $r \approx 7.6 \mu \mathrm{m}$. Note the very close agreement between the size of the three rings in the original image and the simulation.

Such simulations work well when the glory consists of circular colored rings centered on the antisolar point at scattering angle $\theta=180^{\circ}$ (i.e., where the shadow of the observer or aircraft might appear on clouds). Some of the most vivid glories seem to be caused by lenticular clouds (which also produce vivid

$0003-6935 / 08 / 340 \mathrm{H} 25-06 \$ 15.00 / 0$

(C) 2008 Optical Society of America coronas $[3,4])$, but such glories are almost always seen as fragments, typically at the edge of clouds, rather than complete circles. In these cases, there can be considerable uncertainty about the relative position of the antisolar point, and consequently it is very difficult to estimate the size of the water droplets in such clouds.

\section{Estimating the size of droplets}

The sizes of water droplets in clouds can be estimated by comparison of images of circular glories with fullcolor simulations of glories, as shown in Fig. 1(b). Spinhirne and Nakajima [5] reported measurements of the light intensity profiles across the width of a glory made with an airborne scanning radiometer operating at three infrared wavelengths $(740 \mathrm{~nm}$, $2.16 \mu \mathrm{m}$, and $3.74 \mu \mathrm{m}$ ) and concluded that the cloud droplets had a radius of about $4 \mu \mathrm{m}$. Meyer et al. [6] used a similar technique at $753 \mathrm{~nm}$ to determine the maxima of a glory as a function of scattering angle. By comparison with the results of Mie theory calculations, they determined that this particular glory was caused by cloud droplets with an effective radius of $11.3 \mu \mathrm{m}$ and a size distribution with a standard deviation of about $1 \mu \mathrm{m}$.

An even simpler approach is to measure the angular radius of the rings of a glory as recorded in a photograph or digital image and, thus, calculate the radius of water droplets that would cause such rings. Figure 2(a) uses Mie theory simulations to show how the rings of a glory change with the radius 


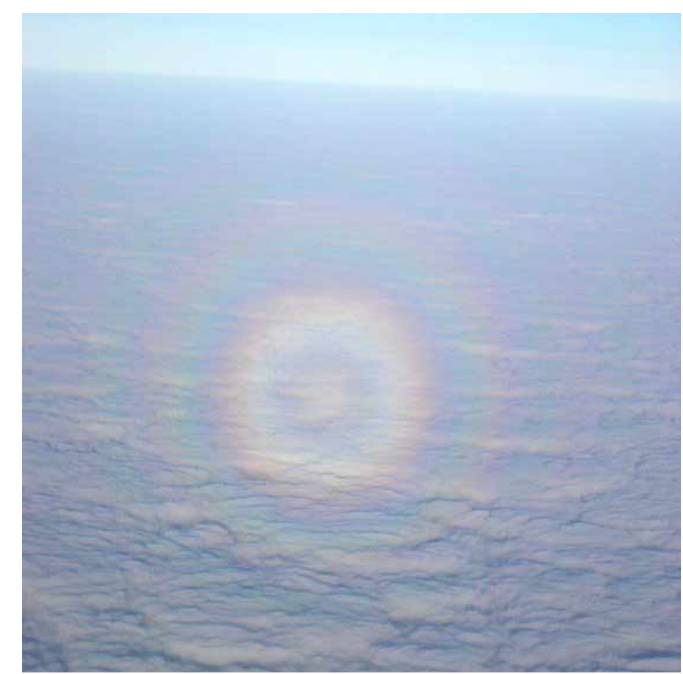

(a)

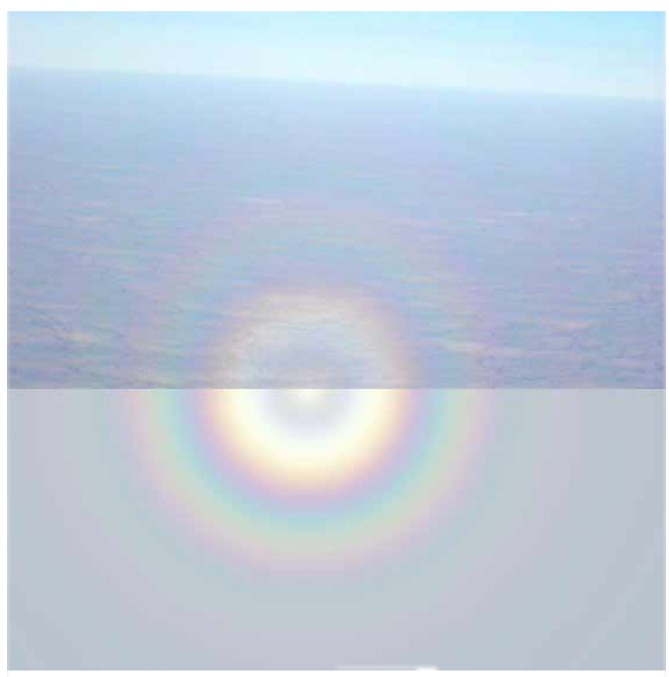

(b)

Fig. 1. (Color online) (a) Image of a circular glory showing three red rings. (b) Comparison with a full color simulation of a glory caused by scattering of sunlight from water droplets with radius $r \approx 7.6 \mu \mathrm{m}$.

$r$ of the water droplets. To a first approximation, the angular radius $R$ of each ring is inversely proportional to the radius $r$ of the water droplets: the four inner red rings appear at radii (measured in degrees) of $R \approx 24 / r, 37 / r, 56 / r$, and $76 / r$ (where $r$ is measured in $\mu \mathrm{m})$. For example, for $r=10 \mu \mathrm{m}$, the innermost red ring would appear at $R \approx 24 / r=2.4^{\circ}$ from the antisolar point, which corresponds to $\theta=$ $180^{\circ}-2.4^{\circ}=177.6^{\circ}$.

A similar approach can also be used to estimate the size of water droplets causing coronas around the sun or moon (i.e., centered on $\theta=0^{\circ}$ ). Figure $2(\mathrm{~b})$ shows how the rings of the corona vary as a function of $r$. As the rings of the corona are much weaker than the direct light near $\theta=0^{\circ}$, the brightness of Fig. 2 (b) has been increased by a factor of 10 so that the top part is "overexposed," thus allowing the colors of the corona to become visible. The three inner red

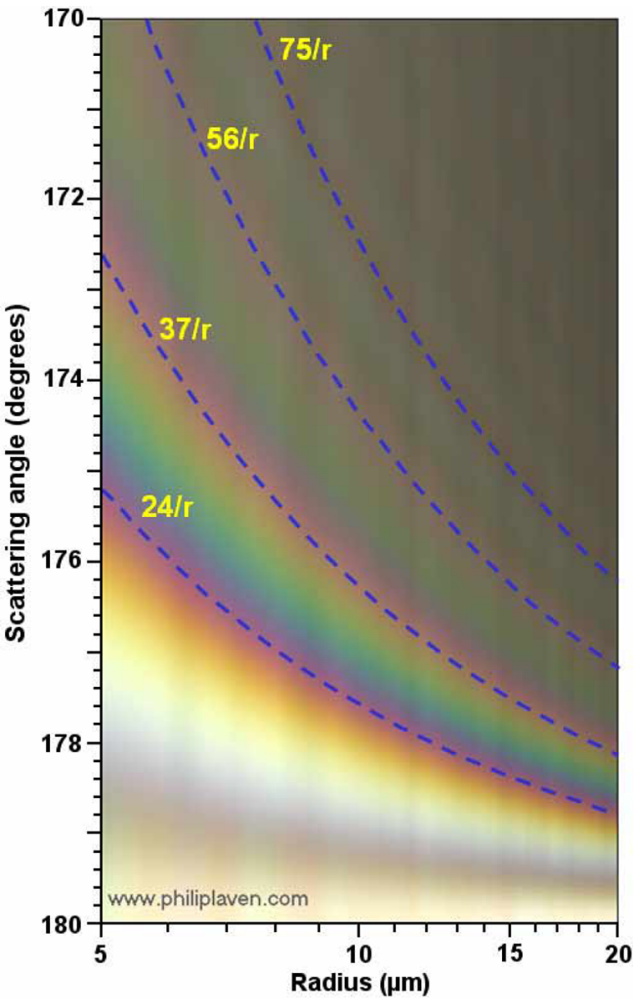

(a)

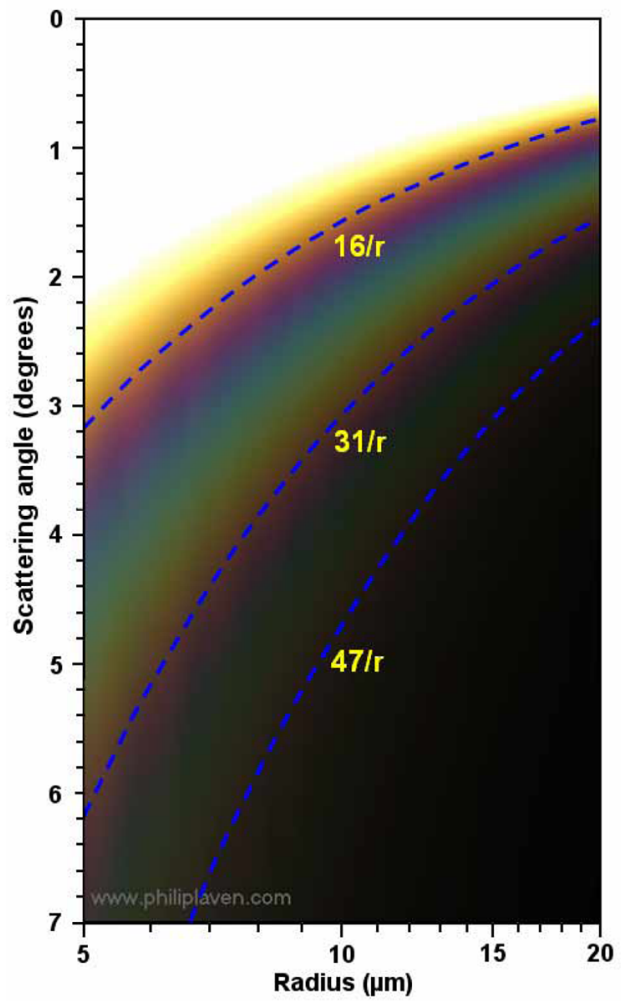

(b)

Fig. 2. (Color online) Mie theory simulations showing (a) the glory and (b) the corona caused by scattering of sunlight by spherical water drops as a function of radius from $r=5 \mu \mathrm{m}$ to $20 \mu \mathrm{m}$. The brightness of (b) has been increased by a factor of 10 to show the rings of the corona. The dotted lines show the red rings of the glory and the corona, with the angular radius $R$ expressed as a function of the radius $r$ of the water droplets. 


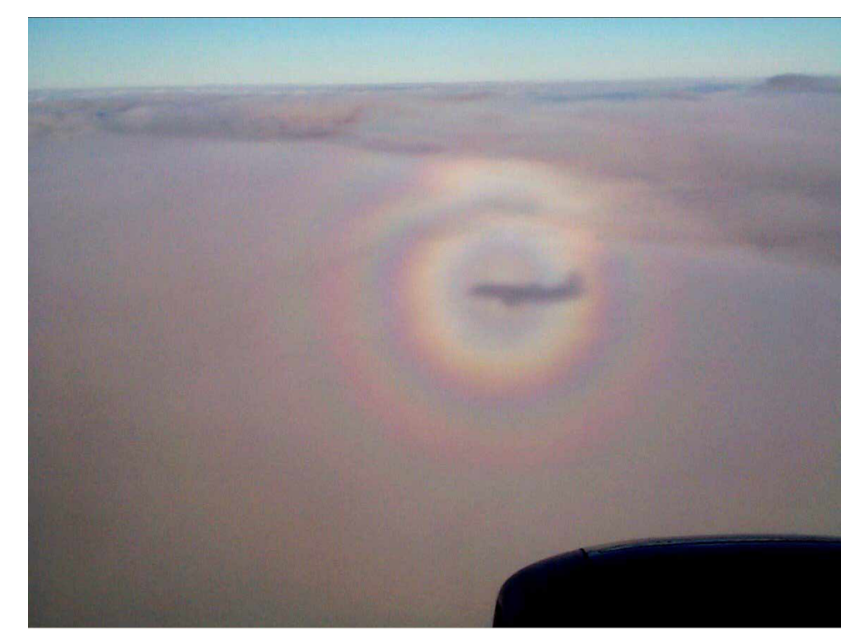

Fig. 3. (Color online) Image of a glory with discontinuous rings caused by backscattering of sunlight from different clouds.

rings of the corona appear at radii (measured in degrees) of $R \approx 16 / r, 31 / r$, and $47 / r$ (where $r$ is measured in $\mu \mathrm{m}$ ) when water droplets are illuminated by sunlight.

\section{Observations of glories}

The glory shown in Fig. 1 is "very circular." In practice, glories are often not circular because they are formed by scattering of light from separate clouds with droplets of different sizes or even from different types of clouds. For example, the lower part of the glory shown in Fig. 3 has uniform circular rings corresponding to droplets of $r \approx 4.9 \mu \mathrm{m}$, whereas the upper part of the glory has rings corresponding to droplets of $r \approx 6.5 \mu \mathrm{m}$.

While most images of glories conform to the expected pattern of colored rings centered on $\theta=180^{\circ}$, some images show glory rings that are definitely not circular. For example, the three images in Figs. 4(a)-4(c) were taken in July 2006 over a period of just $9 \mathrm{~s}$, demonstrating how rapidly the shape of this particular glory was changing (as the aircraft moved, its shadow fell on different clouds). The glory shown in Fig. 4(a) is almost circular with a tendency towards rectangular! On the other hand, the "triangular" glory in Fig. 4(b) has almost straight diagonal lines on either side of the antisolar point. Finally, Fig. 4(c) seems to have rings that are convex towards the antisolar point, rather than being concave as might be expected from "normal" glories.

Figures $\underline{4(\mathrm{~d})}-\underline{4(\mathrm{f})}$ show estimated values of the radius $r$ of the water droplets causing the glories shown in Figs. 4(a)-4(c) respectively-using the $r=24 / R$ relationship where $R$ is the apparent radius of the inner red ring. Note that the estimated values of $r$, particularly in Figs. 4(e) and 4(f), indicate that the bottom of the cloud has smaller droplets than at the top. Although these three images provide very "sparse" data, is it possible that these clouds are "well-ordered" in terms of droplet size? Shaw and Neiman $[3,4]$ suggested that noncircular coronas

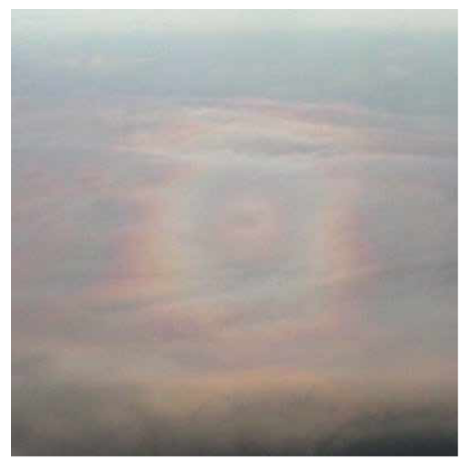

(a)

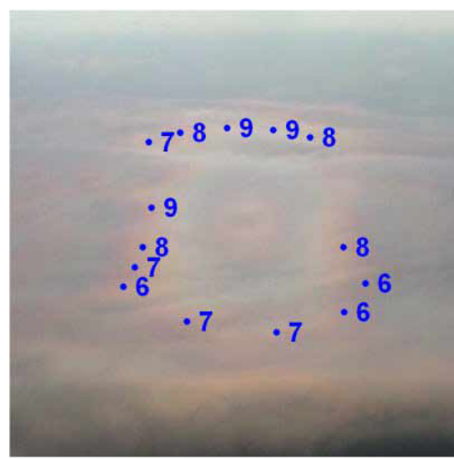

(d)

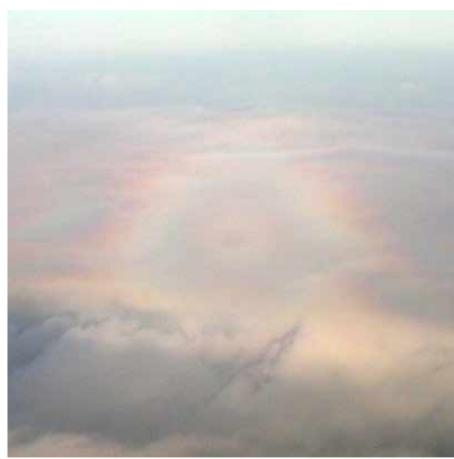

(b)

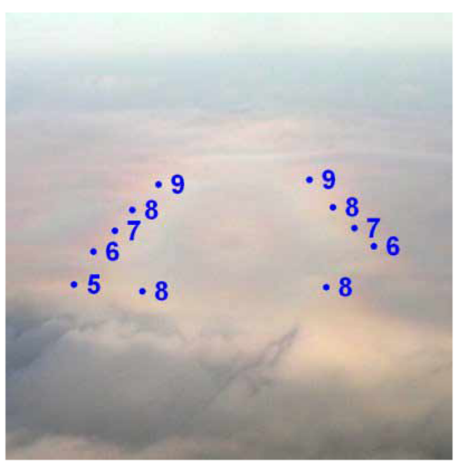

(e)

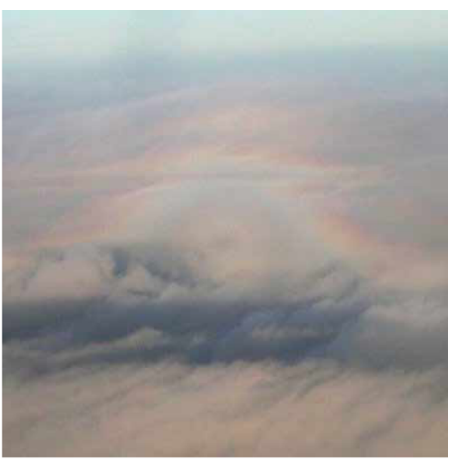

(c)

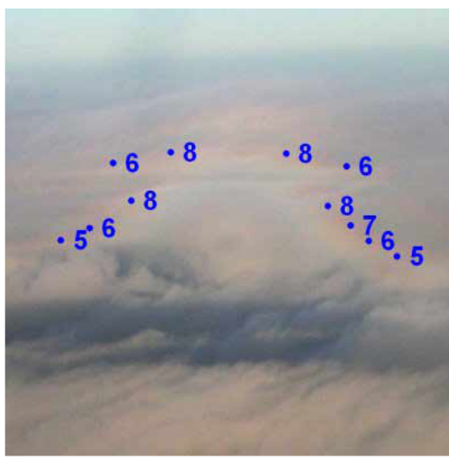

(f)

Fig. 4. (Color online) (a)-(c) Series of images of a glory (taken over a period of $9 \mathrm{~s}$ ) showing significant variations in the shape of the rings. (d)-(f) Estimated values of the radius $r$ (measured in $\mu \mathrm{m}$ ) of the water droplets causing the inner red rings of the glories in (a) -(c). 
on lenticular clouds were caused by variations in droplet size - with smaller droplets at the bottom of such clouds. Brenguier et al. [7] used observations from aircraft flying though clouds to determine the microphysical parameters of the water droplets: the results indicate that marine stratocumulus clouds are vertically stratified with the radius of the droplets increasing with height (i.e., a positive gradient). Rosenfeld et al. [8,9] also reported positive gradients of droplet radius in various types of clouds.

The convex rings of glories shown in Fig. 4(c) are not particularly unusual: once you start looking for them, convex fragments of glory rings seem to appear quite frequently, especially at the edges of clouds.

It is noteworthy that all three glories shown in Fig. 4 seem to have a vertical line of symmetry passing through the antisolar point. How can this symmetry be explained? One possibility is that the water droplets are not spherical: it is well known that large rain drops tend to be flattened as they fall so that their width is greater than their height. As the size of the glory is inversely proportional to the droplet
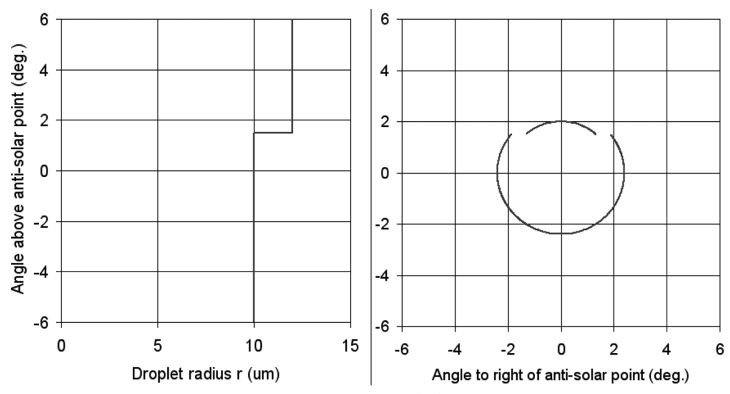

(a)
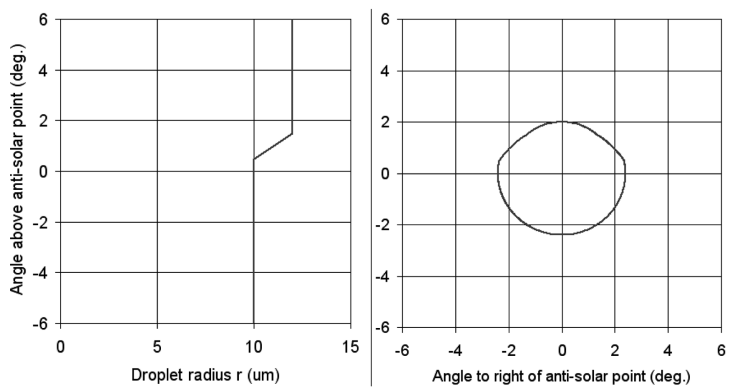

(c)
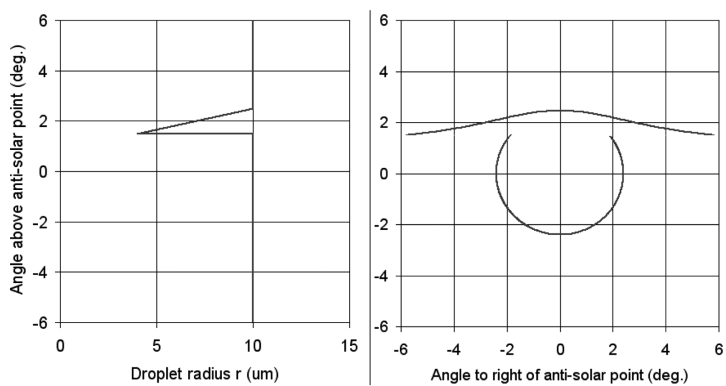

(e) radius, the resulting glory should be elongated in the vertical direction (because the radius of the vertical cross section of flattened droplets is smaller than the radius of the horizontal cross section). Examination of many images of glories has not revealed any examples of significant elongation in the vertical direction. In fact, there are many examples of glories that seem to be compressed in the vertical direction. Even if the droplets were nonspherical, it would be difficult to explain some of the observed features, such as straight or even convex arcs of the rings. In any event, glories are typically caused by very small droplets (e.g., $r<20 \mu \mathrm{m}$ ) for which surface tension ensures that the droplets remain spherical. Thus it seems very unlikely that noncircular glories are due to nonspherical droplets.

Figures 4(d)-4(f) indicate that there are significant variations in the value of $r$ within a single cloudand also suggest that droplets with lower values of $r$ tend to occur at the bottom of clouds. Could these "vertical gradients" in the value of $r$ provide a simple explanation for this vertical symmetry? The effects of
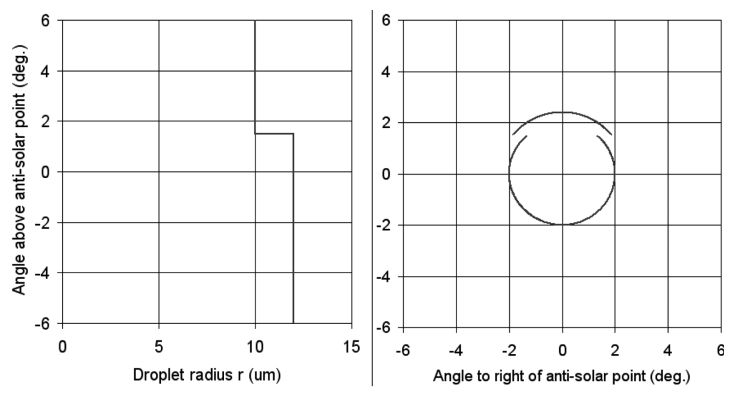

(b)
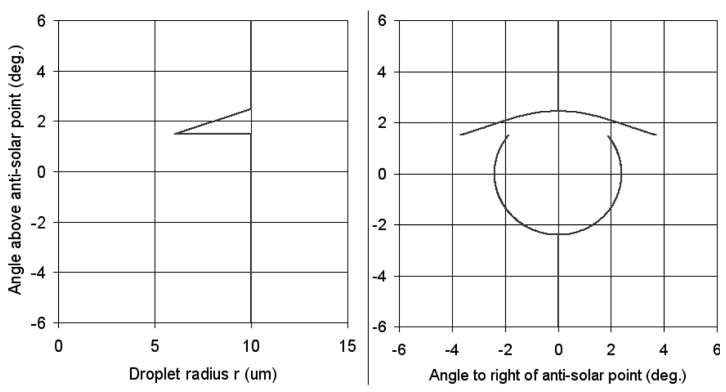

(d)
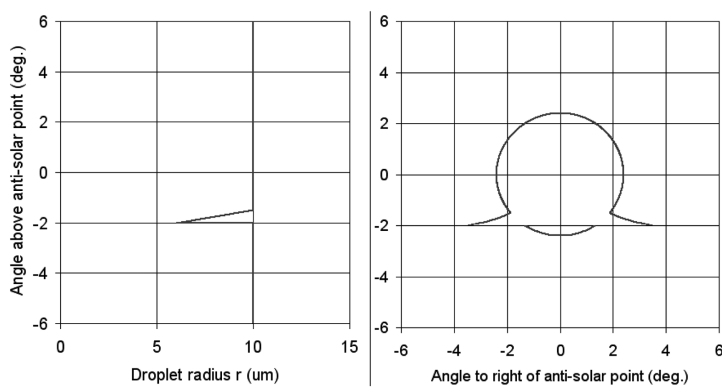

(f)

Fig. 5. Hypothetical vertical profiles of the radius $r$ of water droplets and the calculated shape of the innermost red ring of the resulting glory. 
such vertical gradients in $r$ are explored theoretically in Fig. 5, where each of the diagrams shows a hypothetical vertical profile of $r$ and the resulting shape of the innermost red ring of the glory (as explained in Appendix $\mathrm{A}$, the above-mentioned $R=$ $24 / r$ relationship was used to determine the locus of the glory ring). Figures 5(a) and 5(b) show the effect of a vertical discontinuity in the value of $r$ (i.e., an abrupt transition from $r=12 \mu \mathrm{m}$ to $r=10 \mu \mathrm{m}$, or vice versa). Such discontinuities might seem improbable -but they resemble situations where the upper and lower parts of a glory are caused by backscattering from different clouds (which could easily have different values of $r$ ) - as shown in Fig. 3. Figure 5(c) shows that a gradual reduction in the value of $r$ would straighten part of the innermost ring of the glory. Figures $5(\mathrm{~d})-5(\mathrm{f})$ show that steep gradients in the value of $r$ would result in parts of the ring becoming convex together with "side extensions," which are similar to those shown in Fig. $\underline{6}$ and comparable to the "asymptotic rings" of the corona reported by Shaw and Neiman $[\underline{3}, \underline{4}]$. Careful examination of the glory in Fig. 6 shows that these side extensions coincide with the cliff-like edges of clouds-while Figs. 5(d) and 5(e) suggest that the water droplets are smaller at the bottom of such cliffs than at the top.

Figure 7(a) is one of a remarkable series of images captured in November 2007 by Claudia Hinz from Mount Wendelstein in the Bavarian Alps (Germany). Figure 7(a) has been enhanced to show the details of this glory's unusual rings. Part of the second red ring is V-shaped, marking the transition from a nearcircular fragment of the ring to an extraordinary straight line that very nearly points away from the antisolar point. Figure 7(b) shows estimated values of $r$ calculated using the relationships $r \approx 24 / R$, $37 / R$ and $56 / R$ for the three innermost red rings. The calculated values of $r$ shown in Fig. 7(b) indicate once more that the smallest droplets occur in the lower parts of clouds. As mentioned previously, scattering from lenticular clouds often results in the rings of

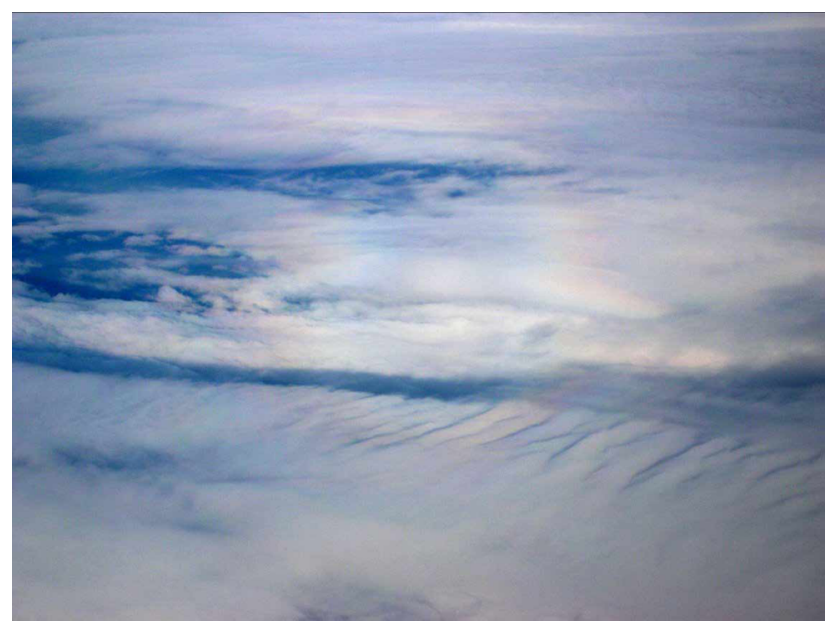

Fig. 6. (Color online) Image of a glory showing "side extensions" to the left and right of the antisolar point [similar to those shown in Fig. 5(f)]. glories appearing to hug the edges of such cloudspresumably because the lower edges of lenticular clouds exhibit steep gradients in the value of $r$, which causes "side extensions" of the glory's rings, as predicted in Fig. 5(e). In the particular case of Fig. 7, the unusual side extensions are due to the orientation of the edge of the cloud.

\section{Conclusions}

Glories are caused by the backscattering of light from spherical water droplets in clouds or fog. The result is usually one or more circular colored rings centered on the antisolar point. However, many glories do not consist of circular rings: this paper includes images of glories with colored rings that, at a first glance, could be described as square or triangular. Furthermore, portions of some rings are convex towards the antisolar point (rather than being concave to the antisolar point).

Comparisons of images of circular glories with fullcolor simulations based on Mie theory can be used to estimate the radius $r$ of the water droplets that cause the backscattering. In the case of noncircular glories, simulations of circular glories are of limited value. However, noting that the four inner red rings of

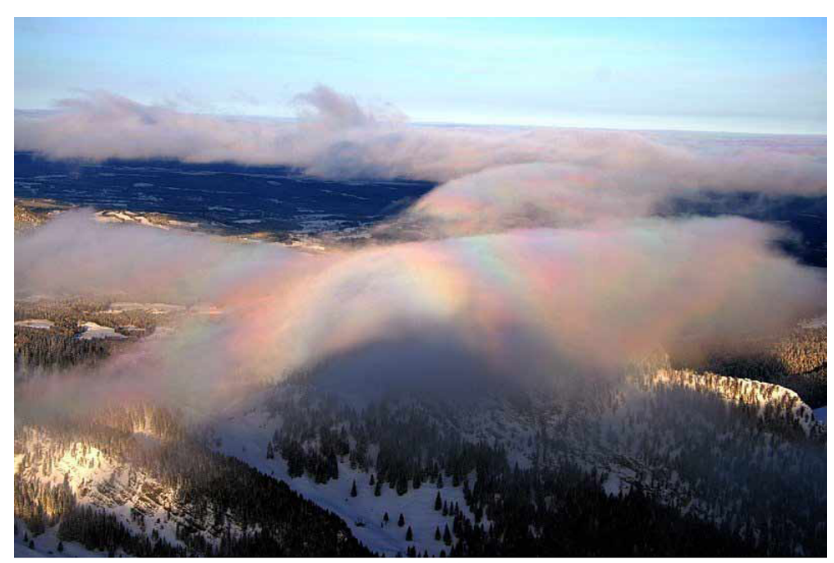

(a)

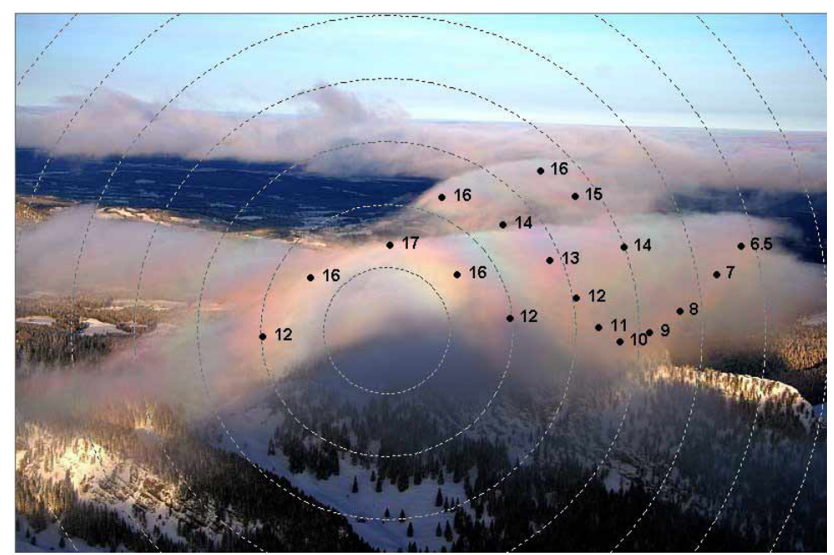

(b)

Fig. 7. (Color online) (a) Image of a very unusual glory observed from Mount Wendelstein (Bavaria, Germany) taken by Claudia Hinz. (b) Estimated values of the radius $r$ (measured in $\mu \mathrm{m}$ ) of the water droplets causing the three red rings of the glory in (a). 
glories appear at radii $R$ (measured in degrees) of approximately $24 / r, 37 / r, 56 / r$, and $76 / r$ (where $r$ is the radius measured in $\mu \mathrm{m}), r$ can be easily estimated by measuring the angular separation between the antisolar point and the red rings of the glory. This simple procedure indicates that the value of $r$ can vary considerably within a given cloud and that the edges of clouds often have steep gradients in the value of $r$ (with smaller droplets at the bottom of clouds).

As the glory is a single scattering phenomenon, the radius $r$ retrieved from a section of the glory is quite "localized" and is, thus, representative of the particular part of the cloud where it is observed. In contrast, other methods based on measurements of reflected radiance at visible or infrared wavelengths must take account of multiple scattering within the cloud, which makes the retrieval of droplet size profiles more difficult. Similarly, values of $r$ inferred from observations of glories are representative of the outer layers of clouds, while the effective radius retrieved from measurements of reflected radiance is averaged over some optical depth inside the cloud.

\section{Appendix A}

Figure 5 gives some examples showing how the shape of the innermost red ring of a glory could be radically affected by hypothetical vertical profiles in the radius $r$ of cloud droplets. This Appendix gives a brief explanation of how these shapes were calculated.

Let $(x, y)$ represent a Cartesian coordinate system, where $x$ represents the horizontal direction and $y$ represents the vertical direction. The origin of this coordinate system, $x=0$ and $y=0$, corresponds to the antisolar point. Assume that the droplet radius $r$ is an arbitrary function of $y$, such as $r=f(y)$. As the radius $R$ of the innermost red ring of a glory is defined by $R=24 / r=24 / f(y)$, all points on this ring must satisfy the equation

$$
x^{2}+y^{2}=(24 / f(y))^{2} .
$$

If $f(y)$ is a constant, Eq. (A1) then becomes $x^{2}+y^{2}=$ $k^{2}$ (where $k$ is a constant), which is immediately re- cognizable as the equation of a circle with radius $k-$ implying that the ring is circular.

If $f(y)$ is a more complicated function, Eq. (A1) can easily be reordered to allow the value of $x$ to be calculated for any given value of $y$ as in Eq. (A2)

$$
x= \pm \sqrt{ }\left((24 / f(y))^{2}-y^{2}\right) .
$$

The diagrams in Fig. 5 represent the locus of points satisfying Eq. (2) for different forms of $f(y)$.

The author would like to thank the two anonymous reviewers for their very helpful and constructive comments.

\section{References}

1. P. Laven, "Simulation of rainbows, coronas, and glories by use of Mie theory," Appl. Opt. 42, 436-444 (2003).

2. P. Laven, "Atmospheric glories: simulations and observations," Appl. Opt. 44, 5667-5674 (2005).

3. J. A. Shaw and P. J. Neiman, "Coronas and iridescence in mountain wave clouds," Appl. Opt. 42, 476-485 (2003).

4. P. J. Neiman and J. A. Shaw, "Coronas and iridescence in mountain wave clouds over Northeastern Colorado," Bull. Amer. Meteor. Soc. 84, 1373-1386 (2003). N. B. This paper is an expanded version of [3] including a discussion of the physics of wave clouds.

5. J. D. Spinhirne and T. Nakajima, "Glory of clouds in the near infrared," Appl. Opt. 33, 4652-4662 (1994).

6. B. Mayer, M. Schröder, R. Preusker, and L. Schüller, "Remote sensing of water cloud droplet size distributions using the backscatter glory: a case study," Atmos. Chem. Phys. 4, 1255-1263 (2004).

7. J-L. Brenguier H. Pawlowska, L. Schüller, R. Preusker, J. Fischer, and Y. Fouquart, "Radiative properties of boundary layer clouds: droplet effective radius versus number concentration," J. Atmos. Sci. 57, 803-821 (2000).

8. D. Rosenfeld and I. M. Lensky, "Satellite-based insights into precipitation formation processes in continental and maritime convective clouds," Bull. Amer. Meteor. Soc. 79, 2457-2476 (1998).

9. D. Rosenfeld and G. Feingold, "Explanations of discrepancies among satellite observations of the aerosol indirect effects," Geophys. Res. Lett. 30, 1776-1779 (2003). 